\title{
The Power of Exercise to Reduce the Risk Factors of Cardiovascular Disease in obese men patients.
}

\author{
Elham Abed Mahdi ${ }^{1}$, Seenaa Kadhum Ali², Haider Fayyadh Hamad Alamiri ${ }^{3}$ \\ ${ }^{1}$ Assistant Prof., Chemistry Department/Faculty of Education for Girl/Kufa University, ${ }^{2}$ Assistant Prof., Chemistry \\ Department/Faculty of Education for Girl/Kufa University, ${ }^{3}$ Assistant Prof., Faculty of Physical Education and \\ Sport Sciences/Kufa University
}

\begin{abstract}
Objective: Firstly To assess the incidence of cardiovascular disease (CVD) risk factors in obese men, and secondly to compare the effects of high intensity interval training (HIIT) and moderate intensity continuous training (MICT) of an 8-week exercise intervention on cardiovascular disease CVD risk factors.

Design and Method: Trails comparing (HIIT) and (MICT) in obese men participants aged 42-52 years and subdivided to two groups: first group consisted from 30 individuals subjected to (HIIT) while the second group comprised from 30 volunteers subjected to (MICT). Participants performed 12 weeks of exercise consisting of $30 \mathrm{~min}$ of exercise five times a week, and all parameters were repeated following the 12-weeks

Result: Both HIIT and MICT elicited significant $(\mathrm{p}<0.05)$ reduction in body mass index (BMI), waist to hip ratio (WHR), metabolic characteristics such as fasting blood glucose (FBG), fasting insulin and lipids variables, as well as improving in insulin sensitivity and blood pressure, in addition to there were significant decline in c- reactive protein (CRP) in study groups after 12- weeks exercise intervention.

The present results show an inverse relationship between the lipoprotein lipase (LPL) activity and body mass index and observed the activity of this enzyme at baseline was less than those in HIIT and MICT, in addition to the activity of this enzyme In individuals with HIIT group was significantly higher $(\mathrm{p}<0.05)$ than the value in those with MICT group.

Conclusion: There is much information about the impact of physical activity on reduction of cardiovascular disease (CVD) risk factors. This information derives from different population groups where sedentary then, physical activity reduced the risk of coronary heart disease and cardiovascular mortality in patients have one or more from CVD risk factors. Present study have shown that reduction in large risk factors CVD can be achieved by moderate or intense physical activity. The risk factors of CVD included: obesity, glucose intolerance, dyslipidemia, and high blood pressure.
\end{abstract}

Keywords: Obesity, Risk Factors of Cardiovascular Diseases, lipoprotein lipase activity, C-reactive protein.

\section{Introduction}

Cardiovascular disease is a wide term, it is used to describe all circumstances affecting the heart and blood vessels, including coronary heart disease, stroke, heart attack and aortic disease. CVD risk factors can be categorized into two classes: invariable and variable ${ }^{1}$. Invariable CVD risk factors are those that cannot be changed. These include age, ethnicity and family history (genetics cannot be changed), among other factors. Variable CVD factors are those that can be lessening or controlled by altered particular habits, by changing certain lifestyle, examples, take off smoking, consumption healthy diet and doing a sport ${ }^{2}$.

Obesity is one of important factors which associated with an increased risk of developing (CVD), particularly heart failure (HF) and coronary heart disease (CHD). The process which explain how obesity increases CVD risk involving the changes in body composition that can alter hemodynamics and effect on heart structure. In addition to adipose tissue produce the Pro-inflammatory 
cytokines which can induce cardiac dysfunction and may be promote the formation of atherosclerotic plaques ${ }^{3}$.

There are several observations from human and animal researches confirming a beneficial role for exercise in the reduction and treatment of CVD. Sedentary life style and obesity/overweight are not only associated with a number of health correlated with risk factors, but are considered to be independent risk factors for CVD, type 2 diabetes mellitus and hypertension. Clinical experiments confirm that lifestyle mediations (dietary modification and increased physical motion) reduce the risk of impaired glucose tolerance and type 2 diabetes ${ }^{4}$. Moreover, epidemiological studies revealed to that risk of hypertension enhancement when a person being overweight. Moderate exercise intensity and frequency have hypotensive effects in physical inactivity hypertensive patients. Long term exercise improves endothelium-dependent dilatation in the aorta and strengthening heart arteries, whereas short-term exercise improves endothelial function in coronary conduit arteries $^{5}$.

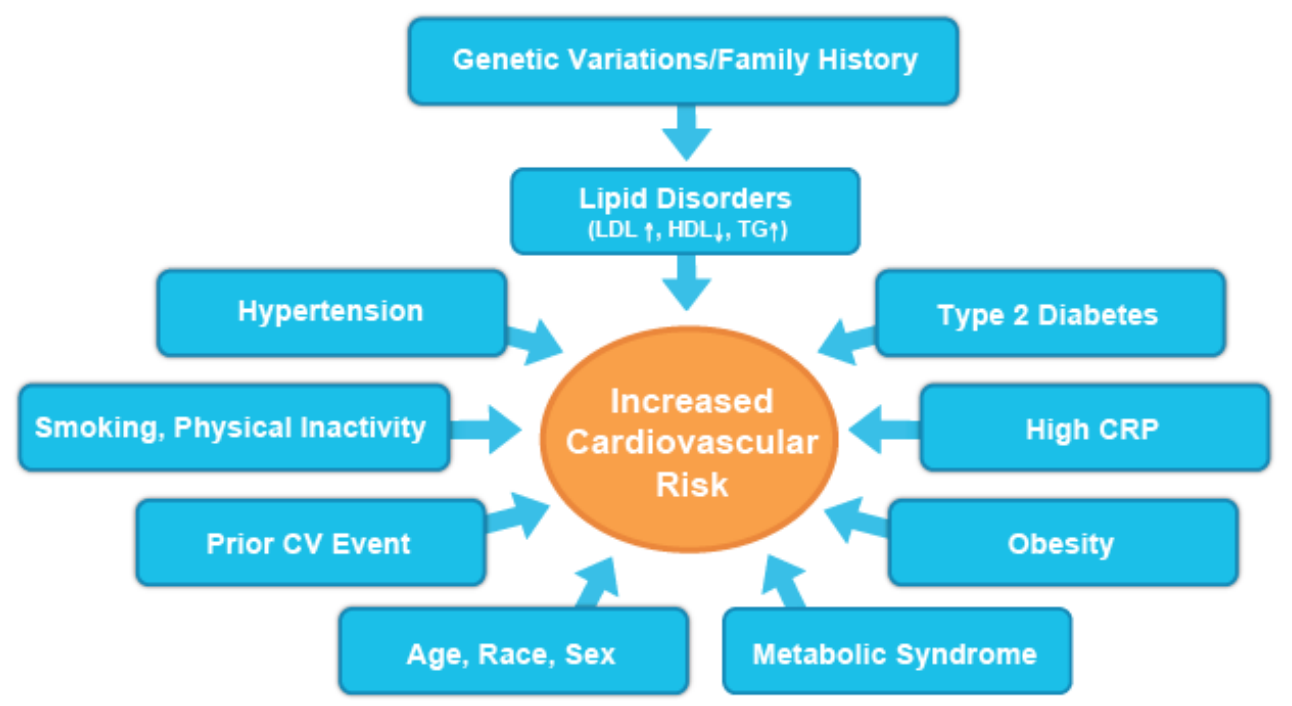

Figure (1): Risk factors for incidence cardiovascular disease (CVD)

In addition to, exercise have been related positively with increasing high density lipoprotein (HDL)cholesterol, and that elucidate why physical activity can have a protective role against coronary heart disease. According to Roussell and Kris-Etherton ${ }^{27}$ changing in life style, physical activity and a healthy diet can raise blood HDL-cholesterol by $10 \%-13 \%$, while training and active motion can independently decrease blood triglyceride concentration ${ }^{27}$.

The aim of the present study was to evaluate whether changes in sedentary lifestyle aspects with special moderate to intense exercise, were effected on the reduce levels of multi CVD risk factors.

\section{Materials and Method}

Study Design: In this study, 60 obese patients (BMI $>30$ ) aged 42-52 years were recruited during the period A pril-July 2019 and subdivided to two groups: first group consisted from 30 individuals subjected to (HIIT) while the second group comprised from 30 volunteers subjected to (MICT).participants were required to take part in an 12-weeks exercise intervention program, and complete a series of physiologic and biochemical test profiles before and after the intervention.

All participants had their height and weight, waist and hip circumference, BP (systolic and diastolic), maximal oxygen volume (VO2 max), total cholesterol, HDL-cholesterol, triglycerides, lipoprotein lipase activity and C- reactive protein levels determined before and following exercise training.

The exercise program for both groups consisted of 12-weeks of high intensity interval training (HIIT) for first group and moderate intensity continuous training (MICT) for second group, two type of exercise consisting of $30 \mathrm{~min}$ of exercise five times a week 
Blood Biochemistry Analysis: All blood sampling was carried out under sterile conditions. Blood glucose measurements which included fasting blood glucose, post prandial blood glucose using certain kits from Spinract, Spain, fasting insulin levels using ELISA kit from Calbiotech, USA then determined HOMA-IR by certain equation. In addition to the measurement of blood lipid profiles were carried out, that included total cholesterol, HDL-cholesterol and triglycerides were obtained between 8 and 10 am following a 10-h fast, using kits from Biolabo, France. Estimates of LDL-cholesterol were calculated using the Friedewald equation. The activity of lipoprotein lipase enzyme and C-reactive protein were determined by applied ELISA method by Elabscience, China kit.

Statistical Analysis: The statistical analysis was achieved by the statistical package for the social science (SPSS) software for windows, version 20.0. the result were represented as mean \pm standard deviation (Mean \pm SD). Paired- sample t test was used to compare variables in same group before and after exercise period and independent- sample $\mathbf{t}$ test was used to compare variables between two groups (HIIT)and (MICT) after exercise period. The confidence interval was set at $95 \%$, thus $\mathrm{p}$ values less than $5 \%(\mathrm{p}<0.05)$ were considered statistically significant.

\section{Results}

A total of 60 participants were classified into two groups based on type of exercise: (HIIT) group $(n=30$, BMI $\left.>30 \mathrm{~kg} / \mathrm{m}^{2}\right)$ and (MICT) group $(\mathrm{n}=30, \mathrm{BMI}>30 \mathrm{~kg} /$ $\mathrm{m}^{2}$ ). The mean values of the anthropometric parameters in both groups before and afterthe period of exercise (12-weeks intervention). pre- vs. post-intervention, statistical analysis of the BMI study persons showed highly significant decreases $(p>0.005)$ when compared pre vs. post exercise intervention in two groups as well as present results pointed out to decline in BMI in (HIIT) group than those in (MICT) group.

The comparison results of WHR in the two groups demonstrated, in the HIIT group, had an average WHR of 0.820 and MICT an average of 0.819 , whilst the two experimental group had significant differences $(\mathrm{p}=0.000)$ in WHR between pre and post training. Systolic and diastolic blood pressure were determined in post and pre training intervention, non-significant increase of systolic blood pressure in post training for both groups (HIIT) and (MICT) in comparison with these values in pre training, whereas, significant reduction were recorded in diastolic blood pressure when compared values in before and following 8- weeks of training, while no such results were noted when the systolic and diastolic blood pressure were tested between (HIIT) and (MICT) groups.

Maximal oxygen uptake is dependent on age and gender, and is a widely used as a predictor tool ofcardiorespiratory fitness. Figure (2) shows the average of VO2 max values for both groups. For pre-exercise, the mean VO2 max for HIIT and MCIT groups was within the poor-fair range of fitness, while, Post the 12-weeks training intervention, the VO2 max values climbed significantly $(\mathrm{p}<0.05)$ for both groups when comparing before and after training. Present study pointed out After 12 weeks of training, the cardiorespiratory fitness of HIIT and MICT groups improved to the fair-good range. Results also showed a main differences between HIIT and MICT, suggesting that MICT had a lower fitness than HIIT.the average of all biochemical variables for both groups before and after the 12-weeks of exercise. A significant decrease in blood sugar levels in pre HIIT and MICT groups comparing with post HIIT and MICT intervention, while did not show significant differences between post HIIT and MICT intervention.

\subsection{8}
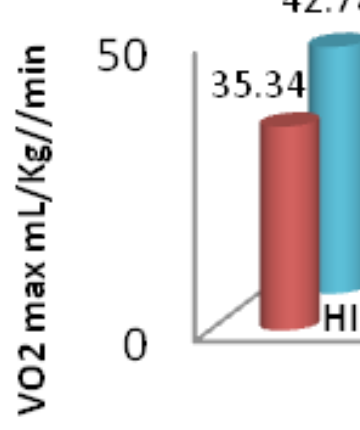

39.87

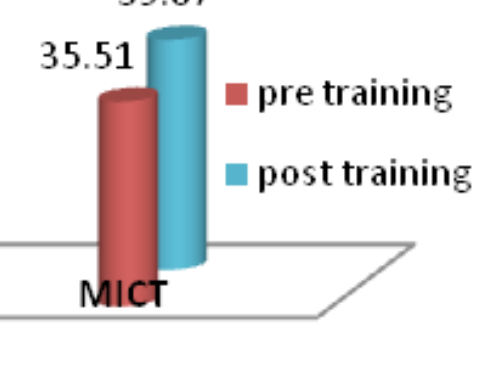

Figure (2): The average VO2 max values measured in HITT and MICT group before and after the 12-weeks training 
In the present study there were significant decreasing of lipoprotein lipase (LPL) in post HIIT and MICT group compared to pre training intervention, no significant were observed when post HIIT and MICT groups compared together $(\mathrm{p}=0.85)$. a significant decrease $(p=0.000)$ of CRP levels in post HIIT and MICT groups when compared with those pre training intervention in both groups.

Fasting insulin level seemed to be significantly reduction $(\mathrm{p}=0.000)$ in the samples of pre HIIT and MICT intervention group comparison to pre training intervention, additionally there were significant variation between HIIT and MICT groups. The insulin resistance level was represented by the HOMA-IR and fasting insulin/glucose ratio (FIGR). The HOMA-IR values in HIIT and MICT groups baseline were $3.009 \pm 1.45$, $3.033 \pm 1.566$; respectively. Results of t-test showed that IR in the HIIT group after exercise intervention was less than those in MICT group, and the differences were statistically significant $(\mathrm{p}<0.05)$. FIGR levels in the post HIIT and MICT groups seemed to be within their levels in the pre HIIT and MICT groups. Results of the present work failed to find significant differences in the levels of FIGR between post training in both groups HIIT and MICT.

The study reported a significant decreasing in the levels of cholesterol, triglyceride, low density lipoprotein binding cholesterol (LDL-C) and very low density lipoprotein binding cholesterol (vLDL-C) after 12 weeks of exercise intervention in HIT and MICT groups when comparing with their levels before exercise intervention, in addition to there were significant variation in these levels of lipid parameters between post HIIT and MCIT intervention. The present study shows that high density lipoprotein binding cholesterol (HDL-C) in serum of post HIIT and MICT groups are significantly increased $(p<0.05)$ compared with that of pre exercise intervention in both groups HIIT and MICT.

\section{Discussion}

This study compared the effects of short-term HIIT and MICT programs on BMI, WHR, $\mathrm{VO}_{2} \mathrm{MAX}$, blood pressure, and several metabolic characteristics, such as fasting blood glucose and insulin in addition to blood lipoprotein levels in sedentary adults. The main findings were that both HIIT and MICT programs significantly improved the BMI, WHR, and diastolic blood pressure However, there were only significantly improved in the HIIT group following 12 weeks of training.
Almost of the participants in present study were obese $(\mathrm{BMI} \geq 30)$ as compared with a large waist circumference characteristic accumulation of the lipid layer in the abdomen (apple pattern), meaning they were classified as obese individuals. Central obesity as a marker of body fat, which can estimated by measuring body mass index (BMI) and waist circumference (WHR) that in turn might effectively predict the risk of metabolic syndrome ${ }^{6}$. Obesity seems to be predominant underlying risk factor not only for the development of metabolic syndrom but also other cardiovascular risk factors ${ }^{7}$. Results of many studies indicated for increasing in body weight and BMI associated with the elevation of ischemic heart disease in several populations ${ }^{21}$. The present finding agreed with the study which mentioned to fact that HIIT has greater effectiveness on weight loss than $\mathrm{MICT}^{11}$, indicating that exercise intensity have efficacy in regulation body composition and local fat exhaustion(Maillard, Pereira et al. 2018). HIIT is superior to MICT in enhancing the secretion of catecholamines, adrenalin, noradernalin, and growth hormone (GH), which elevate fat decomposition(Freda, Shen et al. 2008) to obtain effective weight loss.

Increasing evidences which elucidate that high blood pressure is associating with mortality and CVD. participating in training programs have been recommended as an effective non-medication approach to improve blood pressure, in the same regards, the present study showed that four weeks of HIIT and MICT programs decreased diastolic blood pressure in physically inactive adults. Other study demonstrated a mean reduction from pre- to post-intervention of 3.6 and $3.3 \mathrm{mmHg}$ for resting diastolic bp in the HIIT and MICT intervention with no differences between these programs.

Exercise is an effective method to reduce insulin levels, HIIT intervention was more effective in reduction FBG and insulin resistance compared with MICT training 9 . several studies demonstrated that HIIT can trigger the expression and translocation of glucose transporter 4 (GLUT4) on skeletal muscle cell membrane surfaces more than MICT(Little, Jung etal. 2014). Metcalf et al.(Metcalf, Hosking et al. 2015) observed in a study lasting $6 \sim 19$ years that the regulating and improving insulin resistance will diminishing without physical activity. thus HIIT can promote insulin transportation and insulin tolerance, Ostman et al 2017 reported the lessening in the FBG of patients with insulin resistance syndrome after 16 weeks of continuous training or 
intermittent exercise. Gayda et al.2013 noticed that intermittent exercise is effective in the same way of continuous exercise reduction of FBG in patients with chronic heart failure. Obese individuals suffered from lipid metabolism abnormalities, then leads to raising risk of CVD. Trainings are known as an economic and efficient method to reduce fat accumulation, however, the previous results comparison between HIIT and MICT programs, indicated no significant variations in their effects on TG, TC, LDL, HDL. Other studies revealed no significant difference in the levels of TC, HDL, LDL, triglycerides, and C-reactive protein between HIIT and MICT. The effects of exercise on blood lipid levels in persons with obesity depend on blood lipid levels before training, training intensity, training duration, body fat, calorie intake, metabolic rate and. Several studies have shown that exercise stimulate fat decomposition especially HIIT and MICT are more effective method to reduce TC, as well as the outcomes of this study established that accumulation of LDL inside the blood vessels as a major cause of arteriosclerosis. Our results suggest that trainings especially HIIT help in control on the obesity, reduction the risk factors of CVD, then helping to prevent the development of CVD in obese individual

The main role of lipoprotein lipase is binding to the capillary endothelium of mosttissues, to metabolize triglycerides in circulatingsystem. Free fatty acids produced by this action, thenuptake by peripheral tissues. Most studies report that aerobic exercise increases LPL enzyme expression and activity in postheparinplasma. Another sites of LPL activity include skeletal muscle, where lipolysis produce fatty acids that can be oxidized as an energy source, and adipose tissue, and adipose tissue where fatty acids released from circulating lipoproteins by LPL are reasterfied and stored as triglycerides. The Investigators in animal have generally identified increases in skeletal muscle LPL activity and decreases in adipose LPL with exercise, but human studies are indicated that exercise promote increases in LPL activity in both tissues.Muscle is the main place of triglyceride removal in humans, and if LPL is central to this process, LPL-stimulate lipolysis in muscle, wherefore, may be a basic factor in the generation of high-density lipoproteincholesterol (HDL-C) . In addition to, the catabolism of triglyceride-rich lipoproteins, raising the generation of HDL-C and HDL2-C in exercising human muscle. There are much evidences that fasting lipid alterations occur in response to exercise due to the stimulate of adipose tissue LPL activity, which correlated with weight loss, so in the morbid obese, weight loss is combined with increased LPL expression in adipose tissue.

A metabolic abnormalities in obesity patients associated with increase acute-phase response. C-reactive protein (CRP), is the important component of acute-phase proteins and considered a sensitive and major marker of bacterial infection, physical tissue damage, and other inflammatory conditions. CRP is controlled by the proinflammatorycytokines including interleukin-1 (IL-1), tumor necrosis factor-a (TNF-a), and interleukin-6 (IL-6), which produced by hepatocytes. Recent researches provide a proof that inflammation might play a role in the incidence of cardiovascular disease, CRP with other inflammatory markers being regarded as indicators of atherothrombotic disease.

\section{Conclusions}

There is much information about the impact of physical activity on reduction of cardiovascular disease (CVD) risk factors. This information derives from different population groups where sedentary then, physical activity reduced the risk of coronary heart disease and cardiovascular mortality in patients have one or more from CVD risk factors. Present study have shown that reduction in large risk factors CVD can be achieved by moderate (MICT) or intense (HIIT) physical activity. The risk factors of CVD included: obesity, glucose intolerance, dyslipidemia, and high blood pressure all of these parameters improving in good state. In addition to, the present research demonstrated that physical achievement is inversely correlated with levels of LPL and inflammatory marker (CRP) in obese patients and improve this parameters values after exercise programs.

Financial Disclosure: There is no financial disclosure.

Conflict of Interest: None to declare.

Ethical Clearance: All experimental protocols were approved under the Chemistry Department and all experiments were carried out in accordance with approved guidelines.

\section{References}

1. Ghonaem, SE, M. Effectiveness of planned discharge instructions on patients' recovery following coronary artery bypass graft surgery." 
IOSRJNHS.2018; 7(6): 8-16.

2. Mesiäinen, N, E Pirttimäki G. Kosonen (. "Middleaged patient's experiences of pain management after cardiac surgery."

3. Carbone, S., J. M. Canada, H. E. Billingsley, M. S. Siddiqui, A. Elagizi and C. J. Lavie. «Obesity paradox in cardiovascular disease: where do we stand?» Vascular Health and Risk Management 2019;15: 89.

4. Scrutinio, D, F Bellotto, R Lagioia A. Passantino. «Physical activity for coronary heart disease: cardioprotective mechanisms and effects on prognosis.» Monaldi archives for chest disease $2005 ; 64: 2$.

5. Linke, A, S Erbs R. Hambrecht. "Exercise and the coronary circulation - alterations and adaptations in coronary artery disease." Progress in cardiovascular diseases 2006;48(4): 270-284.

6. Al-Lawati, J A, P Jousilahti. "Body mass index, waist circumference and waist-to-hip ratio cut-off points for categorisation of obesity among Omani Arabs." Public health nutrition 2008; 11(1): 102108.

7. Beydoun, M A, M Kuczmarski, Y. Wang,. «Receiver-operating characteristics of adiposity for metabolic syndrome: the Healthy Aging in Neighborhoods of Diversity across the Life Span (HANDLS) study.» Public Health Nutrition 2011; 14(1): 77-92.

8. Bi, X, Y Loo, S Ponnalagu,CJ. Henry. «Obesity is an independent determinant of elevated C-reactive protein in healthy women but not men.» Biomarkers 2019; 24(1): 64-69.

9. Cassidy, S, C Thoma, D Houghton, M Trenell. "High-intensity interval training: a review of its impact on glucose control and cardiometabolic health." Diabetologia 2017; 60(1): 7-23.

10. Chang, H Chen, HH. "Which obesity index is the best predictor for high cardiovascular disease risk in middle-aged and elderly population?" Archives of gerontology and geriatrics 2018; 78: 165-170.

11. Cheema, B, T Davies, M Stewart, S. Papalia). "The feasibility and effectiveness of high-intensity boxing trainingversus moderate-intensity brisk walking in adults with abdominal obesity: a pilot study." BMC sports science, medicine and rehabilitation 2015; 7(1): $1-10$.
12. Costa, E, J Hay, D Kehler.”Effects of high-intensity interval training versus moderate-intensity continuous training on blood pressure in adults with pre-to established hypertension: a systematic review and meta-analysis of randomized trials." Sports Medicine 2018; 48(9): 2127-2142.

13. Durstine, J,E Anderson, R Porter, X. Wang.Physical activity, exercise, and lipids and lipoproteins. Cardiorespiratory fitness in cardiometabolic diseases, Springer: 2019; 265-293.

14. Freda, P. U., W. Shen, S. B. Heymsfield.”Lower visceral and subcutaneous but higher intermuscular adipose tissue depots in patients with growth hormone and insulin-like growth factor I excess due to acromegaly." The Journal of Clinical Endocrinology \& Metabolism 2008; 93(6): 23342343.

15. Gayda, M, E Normandin, P Meyer, ."Comparison of carbohydrate and lipid oxidation during continuous and intermittent exercise in patients with chronic heart failure." CanadianJournal of Cardiology 2013; 29(8): 990-992.

16. He, P, T Jiang, X. OuYang. «Lipoprotein lipase: Biosynthesis, regulatory factors, and its role in atherosclerosis and other diseases.» Clinica Chimica Acta 2018; 480: 126-137.

17. Helgerud, J, K Høydal, E. Wang, T.Aerobic highintensity intervals improve $\mathrm{V}^{\cdot} \mathrm{O} 2 \mathrm{max}$ more than moderate training.» Medicine \& Science in Sports \& Exercise 2007; 39(4): 665-671.

18. James, PA, S Oparil, B Carter, W Cushman. “2014 evidence-based guideline for the management of high blood pressure in adults: report from the panel membersappointed to the Eighth Joint National Committee (JNC 8)." Jama 2014; 311(5): 507-520.

19. Katzmarzyk, P, T Church. "Cardiorespiratory fitness attenuates the effects of the metabolic syndrome on all-cause and cardiovascular disease mortality in men." Archives of internal medicine 2004; 164(10): 1092-1097.

20. Kim, SW, YK Jang, JEKwon. "Correlation between Work Related Physical Activity and Serum Lipid: The Korean National Health and Nutrition Examination Survey 2013-2015.” Korean Journal of Family Practice 2017; 7(5): 777-780.

21. Lamon-Fava, S, P Wilson, E Schaefer "Impact of body mass index on coronary heart disease risk factors in men and women: the Framingham 
Offspring Study.” Arteriosclerosis, thrombosis, and vascular biology 1996, 16(12): 1509-1515.

22. Litleskare, S, E Enoksen, M Sandvei. "Sprint Interval Running and Continuous Running Produce Training Specific Adaptations, Despite a Similar Improvement of Aerobic Endurance Capacity-A Randomized Trial of Healthy Adults." International Journal of Environmental Research and Public Health2020; 17(11): 3865.

23. Little, J, M Jung, AE Wright. "Effects of highintensity interval exercise versus continuous moderate-intensity exercise on postprandial glycemic control assessed by continuous glucose monitoring in obese adults." Applied physiology, nutrition, and metabolism 2000; 39(7): 835-841.

24. MacInnis, M, M Gibala. "Physiological adaptations to interval training and the role of exercise intensity.” The Journal of physiology 2017; 595(9): 2915-2930.

25. Mahadik, S, S Deo, SD Mehtalia. "Association of adiposity, inflammation and atherosclerosis: the role of adipocytokines and CRP in Asian Indian subjects." Metabolic syndrome and related disorders 2000; 6(2): 121-128.

26. Ostman, C, N Smart, D Morcos. "The effect of exercise training on clinical outcomes in patients with the metabolic syndrome: a systematic review and meta-analysis." Cardiovascular diabetology 2017; 16(1): 110.

27. Roussell, M, P Kris-Etherton. "Effects of lifestyle interventions on high-density lipoprotein cholesterol levels." Journal of Clinical Lipidology 2007; 1(1): 65-73. 